\title{
Development of Optically-Driven Metallic Microrotors Using Two-Photon Microfabrication
}

\author{
Takashi IKEGAMI ${ }^{* 1}$, Ryota OZAWA ${ }^{* 1}$, Michael. P. STOCKER ${ }^{* 2}$, Kathleen MONACO ${ }^{* 2}$, John T. FOURKAS ${ }^{* 2,3}$ and Shoji \\ MARUO $^{* 1}$ \\ ${ }^{* 1}$ Department of Mechanical Engineering, \\ Yokohama National University, 79-5 Tokiwadai, Hodogaya-ku, Yokohama 240-8501, Japan \\ ${ }^{* 2}$ Department of Chemistry and Biochemistry, University of Maryland, \\ College Park, MD 20742-4454, U.S.A \\ ${ }^{* 3}$ Institute for Physical Science and Technology, University of Maryland, College Park, MD $20742-$ \\ 4454, U.S.A \\ E-mail: Ikegami-takashi-rg@ynu.ac.jp
}

\begin{abstract}
We have developed metallized, cross-shaped microrotors driven by scanning a low-power laser beam. The metallized microrotors were fabricated by the combination of two-photon microfabrication, electroless plating and laser ablation. It is demonstrated that a cross-shaped, metallized microrotor can be driven at a laser power of $3 \mathrm{~mW}$. We also analyze the radiation pressure exerted on the blade to investigate the driving mechanism of the metallized micorotor. As a result, it was confirmed that a tilted blade could generate larger torque than a horizontal blade due to the reflection of an incident Gaussian laser beam. Such optically driven metallized micromachines will be useful for low-cost, functional lab-on-a-chip devices.

Keywords: Two-photon microfabrication, Electroless copper plating, Laser ablation, Opticallydriven metallic microrotor
\end{abstract}

\section{Introduction}

DOI:10.2961/jlmn.2013.01.0002

Optical tweezers using a focused laser beam have been widely employed to manipulate micro/nano scale samples such as micro/nano particles, cells and DNA molecules [14]. Since optical tweezers enable us to manipulate microobjects in a sealed liquid environment, they can be also used in microfluidic devices including micropumps, microvalves and flow sensors using optically driven colloidal particles [5-7]. Laser direct writing, such as twophoton microfabrication $[8,9]$, allows for the fabrication of sophisticated, three-dimensional microfluidic devices such as lobed micropumps and viscous micropumps [10-12]. Microtweezers and microvalves have been also developed $[13,14]$. Optically driven micromachines have several advantages. For example, built-in micromachines can offer precise control of microfluidics without external pumps and valves. Polymeric microfluidic devices are also suitable for disposable usage for medical diagnostics.

However, the optically driven micromachines have some drawbacks for practical use. One of the main disadvantages is the requirement of a laser whose average power ranges from several $100 \mathrm{~mW}$ to $1 \mathrm{~W}$ to drive multiple micromachines $[10,11]$. This high power requirement arises because most of optically driven micromachines developed previously were made of a transparent photopolymer or glass, so the optical net force exerted on them is attractive. Thus, an expensive and relatively large driving system is required, even though the microfluidic circuits containing micromachines are inexpensive and highly functional.

As a promising approach to reduce the laser power required for driving micromachines, we metallized movable microcomponents by electroless plating. When the micromachines are metallized, a focused laser beam reflected from their surface generates a repulsive force. The repulsive force due to the reflection at the metallic surface is larger than the attractive force exerted on transparent dielectric structures. In addition, since most of the repulsive force contributes to push the micromachines, the net repulsive force is large enough to drive them at a low laser power. Although it has been demonstrated that metallic particles can be manipulated by a laser beam [15-17], metallized micromachines driven by light have not been reported previously to our knowledge.

Here, we report a cross-shaped, metallized microrotor driven by a low-power laser on the order of $\mathrm{mW}$. The metallized microrotor was developed by the combination of electroless plating and two-photon microfabrication [18] The fabrication process and testing of the metallized micromachines are described in detail. In addition, the driving mechanism of the cross-shaped metallized microrotor is addressed by analyzing the radiation pressure exerted on the blade of the metallized microrotor.

2. Fabrication of metallized micromachines by twophoton microfabrication combined with electroless plating and laser ablation

\subsection{Fabrication process of a metallized microrotor}

To produce metallized movable microparts, we employed a fabrication process based on two-photon microfabrication with subsequent electroless plating and laser ablation, as was demonstrated in a previous report [18]. Fig. 1 illustrates the fabrication process of a metallized movable micropart. The detail of the process is 


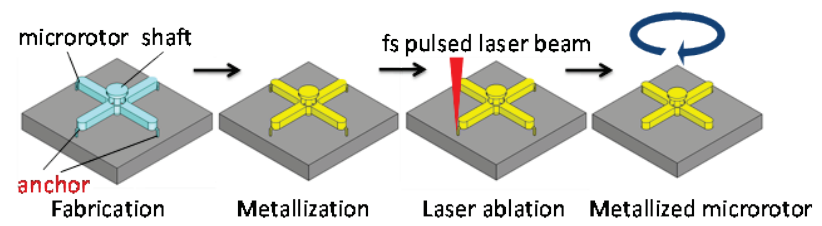

Fig.1 Fabrication process of metallized movable microparts. The movable microparts supported with anchors are fabricated by two-photon microfabrication. After subsequent electroless plating, the anchors are removed by laser ablation.

as follows: First, a microrotor supported with anchors is fabricated on a glass substrate by two-photon microfabrication. The movable micropart is then metallized by electroless copper plating $[18,19]$. Since the movable micropart is suspended by the anchors, electroless plating is performed on the entire movable micropart. Finally, the anchors are removed from the movable micropart by laser ablation with a femtosecond pulsed laser beam. As a result, 3D metallized movable microparts can be fabricated. The combination of the anchor supporting method and laser ablation enables stable, high-precision fabrication of relatively large movable microparts with uniform metallization.

\subsection{Preparation of acrylic resin}

To prepare an acrylic resin suitable for electroless copper plating, we mixed two kinds of acrylic monomers, tris (2hydroxyethyl) isocyanurate triacrylate (Sartomer: SR-368) and ethoxylated (6) trimethylolpropane triacrylate (Sartomer: SR-499) with a weight ratio of 1:1. As a photoinitiator, we used Lucirin TPO-L (ethyl-2,4,6trimethylbenzoylphenylphosphinate), which has a high two-photon-initiated polymerization yield rate at a wavelength of $800 \mathrm{~nm}$ [20]. The concentration of Lucirin TPO-L (BASF) was 3 wt.\%.

\subsection{Metallization of a cross-shaped microrotor produced by two-photon microfabrication}

A polymeric microrotor with anchors was fabricated by a two-photon microfabrication system that we developed [10]. The microrotor was metallized by electroless copper plating with optimal conditions described in detail in a previous report [18].

Figs. 2(a) and 2(b) show a scanning electron microscope (SEM) image and an optical image, respectively, of a cross-shaped metallized microrotor with anchors. The microrotor (diameter: $60 \mu \mathrm{m}$ ) is supported by four anchors (length: $2 \mu \mathrm{m}$, height: $6 \mu \mathrm{m}$ ) attached at the tip of the rotor blade. The cross section of each anchor is a square, $1 \mu \mathrm{m}$ on a side. Fig. 2(c) shows a transmission electron microscope (TEM) image of the copper layer deposited on the polymeric structure. To observe the cross section of the copper layer, we first prepare a polymeric plate made from the same acrylic resin by UV exposure. And then a 5$\mathrm{mm}$-square thin layer was fabricated on the polymer plate by two-photon microfabrication. The polymeric plate with the 5-mm-square thin layer was embedded in epoxy resin and then the sample was sliced with a microtome. The sliced sample was observed by TEM. The average thickness of the copper layer was approximately $324 \mathrm{~nm}$.

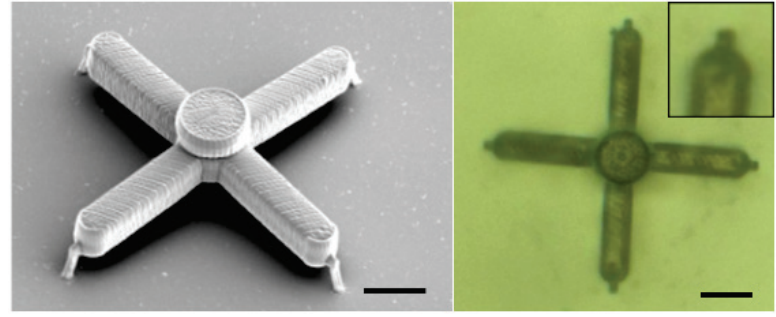

(a)

(b)

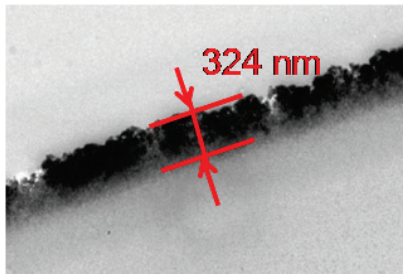

(c)

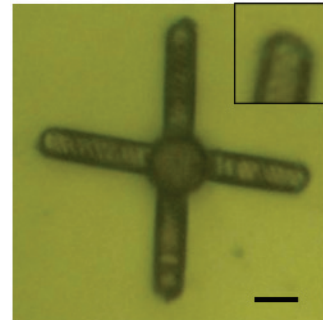

(d)
Fig. 2 Metallization of a cross-shaped microrotor supported by anchors. (a) SEM image of a polymeric microrotor. The scale bar is $10 \mu \mathrm{m}$. (b) Optical image of the same metallized microrotor before laser ablation. The scale bar is $10 \mu \mathrm{m}$. (c) TEM image of copper layer on polymeric structure. (d) Optical image of a metallized microrotor after laser ablation. The scale bar is $10 \mu \mathrm{m}$.

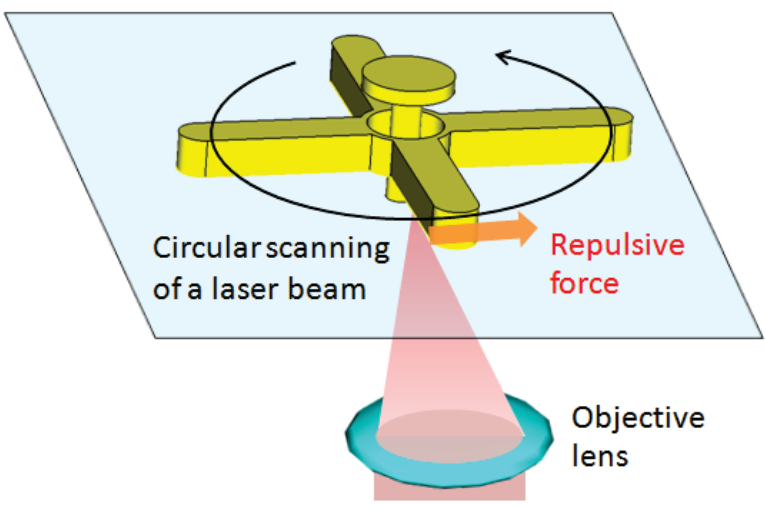

Fig. 3 Optical driving of a metallized microrotor by scanning a laser beam.

Due to the use of the anchor supporting method, the microrotor was completely metallized by the electroless copper plating.

\subsection{Removal of anchors by laser ablation}

After metallization, we removed the anchors by laser ablation with a femtosecond pulsed laser (Mai-Tai, Spectra Physics Inc., wavelength $750 \mathrm{~nm}$; repetition rate $80 \mathrm{MHz}$; pulse width $100 \mathrm{fs}$ ). The laser beam was focused on the anchor using an objective lens with a numerical aperture of 0.65 .

In a previous report, we explored the optimal exposure conditions to remove the anchors with minimal defects and 
minimal excess cutting of its rotor blade [18]. In the optimal experimental conditions, the exposure time for each laser ablation was $0.5 \mathrm{~s}$ at a laser power was $150 \mathrm{~mW}$. The laser beam was focused at the bottom of the anchor to remove the anchors completely. We were able to remove the anchor of the metallized cross-shaped microrotor without deformation using this method, as shown in Fig. 2(d). The scattered resin remaining after laser ablation could be removed easily by washing with ethanol.

\section{Optical actuation of a metalized, cross-shaped microrotor with a low-power laser beam}

We tested driving a metallized cross-shaped microrotor by an optical micromanipulation technique using a scanning $\mathrm{HeNe}$ laser beam (wavelength: $632.8 \mathrm{~nm}$; maximum output power: $30 \mathrm{~mW}$ ). Fig. 3 shows the schematic diagram of driving of a metallized microrotor. In our method, a laser beam is scanned circularly to apply optical torque to the blades. Each blade is pushed along a circular trajectory as the laser beam passes through it, so that the microrotor can be rotated. The power of the laser beam was adjusted with a variable neutral density filter. The laser beam was focused by an objective lens (NA: 0.4) and scanned by galvo mirrors (GSI lumonics, M2 scanner). The diameter of the circular trajectory of the scanning laser beam was $50 \mu \mathrm{m}$, which is smaller than the diameter of the microrotor. The surrounding liquid was glycol ether ester. Fig. 4 shows sequential images of a rotating metallized microrotor taken by a high-speed camera with a frame rate of $250 \mathrm{fps}$. The laser power through the objective lens was adjusted to $3 \mathrm{~mW}$ for the experiments shown here. From the sequential images taken by the high-speed camera, we confirmed that each blade was pushed by repulsive force exerted on its edge when the laser beam passed through the blade. As a result, the rotor could be rotated by repetitive pushing.

Fig. 5 shows the dependence of the rotation speed of the microrotor on the scanning speed of a laser beam. These results indicate that the rotation speed of the microrotor is proportional to the scanning speed of the laser beam. The maximum rotation speed achieved was approximately $86 \mathrm{rpm}$ at a laser scanning speed of $600 \mathrm{rpm}$.

In contrast, most previously developed photopolymer micromachines have been driven by attractive forces exerted on an optically trapped portion of the micromachine [10-14]. Although attractive forces can offer precise position and velocity control of the micromachines, a laser beam whose average power ranges from 0.5 to $1 \mathrm{~W}$ is required to drive the micromachines because the attractive force is relatively small. On the other hand, the metallized micromachines demonstrated here can be driven with a laser power of $3 \mathrm{~mW}$ owing to the relatively large repulsive force.

\section{Analysis of the repulsive driving mechanism of the metallized microrotor}

Fig. 6 shows a snapshot of a rotating microrotor. In this image, it is seen that the length of the crossed blades appear different in two directions. From this experimental observation, it was found that the metallized cross-shaped
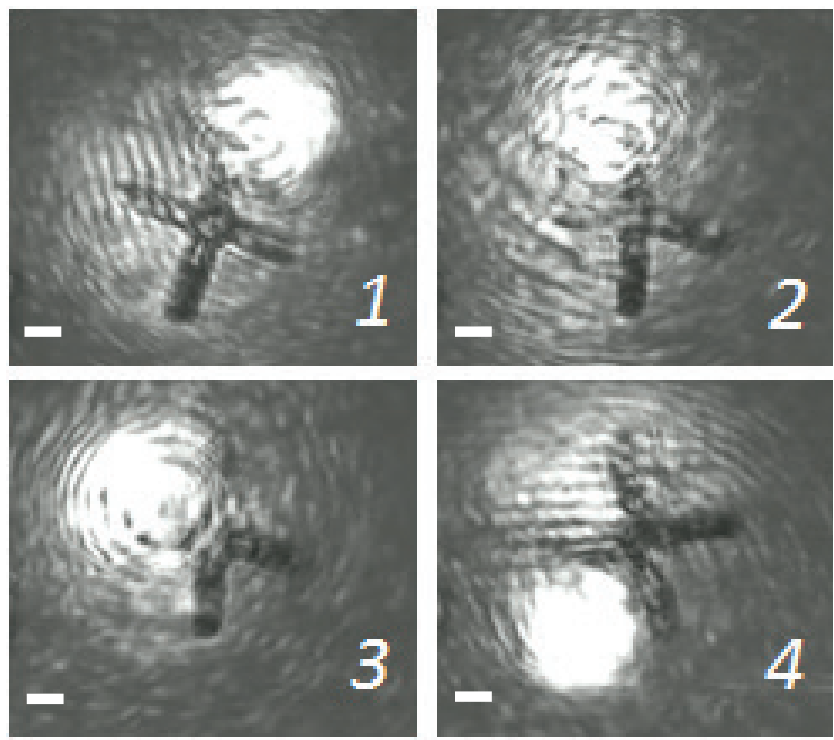

Fig. 4 Sequential optical images of a rotating microrotor. The scale bars are $10 \mu \mathrm{m}$

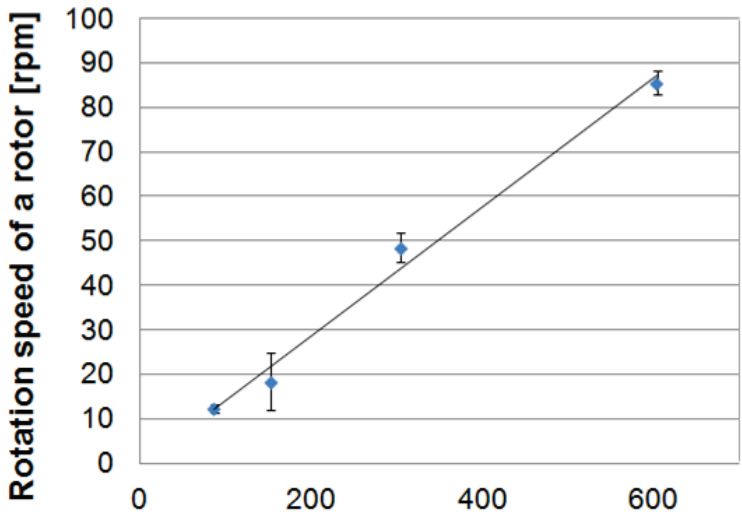

Scanning speed of a laser beam [rpm]

Fig. 5 Rotation speed of a metallized microrotor vs. scanning speed of a laser beam. The laser power was $3 \mathrm{~mW}$.

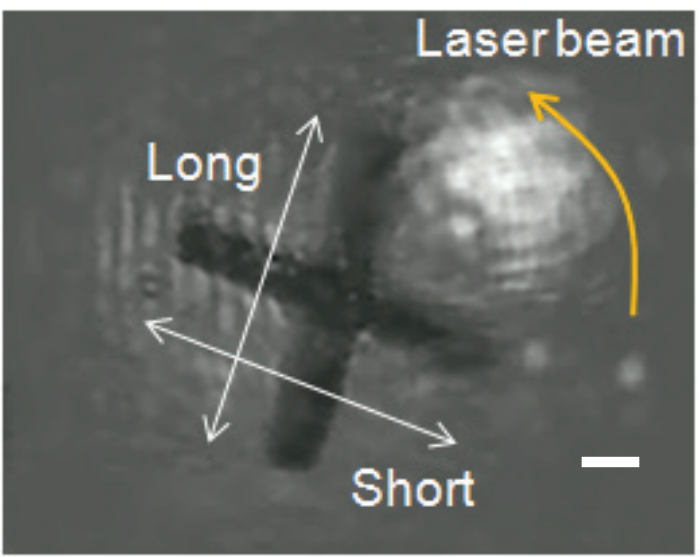

Fig. 6 A snapshot of a tilted microrotor during rotation. The scale bar is $10 \mu \mathrm{m}$

microrotor was tilted during rotation. The maximum tilt angle was estimated to be $15^{\circ}$ according to the geometric constraint of the design of the microrotor. Based on this tilt, we believe that the driving mechanism is the one shown in Fig 7. First, the blade is pushed up when the laser beam 


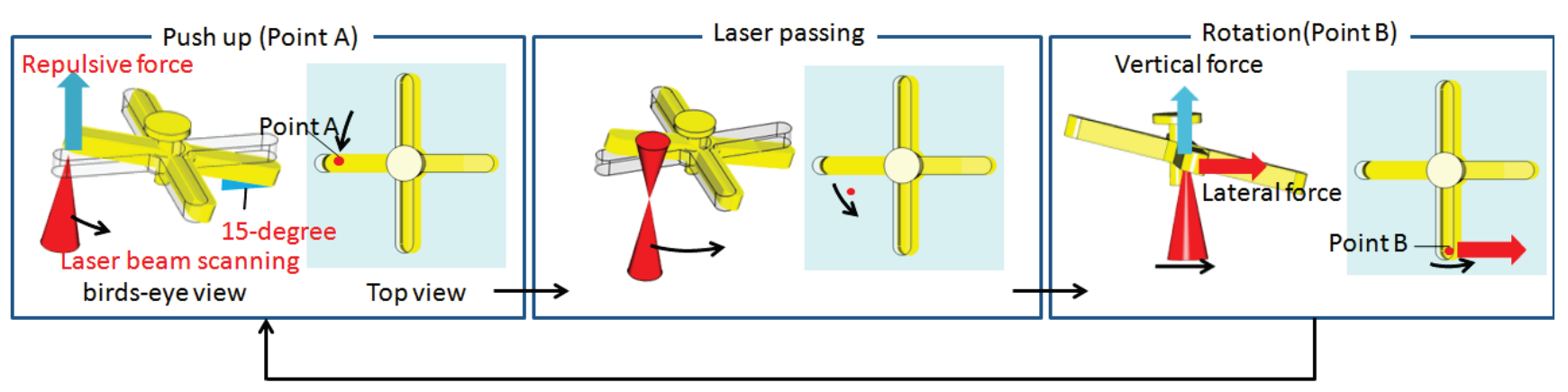

Fig. 7 Driving mechanism of a metallized microrotor. The metallized microrotor is tilted at $15^{\circ}$ to the glass substrate during rotation.

reaches Point $\mathrm{A}$, and the microrotor is tilted. The laser beam then reaches Point $\mathrm{B}$ while the microrotor is still tilted, because the scanning speed of the laser beam is fast enough the microrotor keeps floating in the liquid. Therefore, the microrotor is pushed both laterally and vertically when the laser beam is reflected from the bottom face of the tilted blade. This sequence is repeated at each blade, so that the microrotor is intermittently rotated by relatively large optical torque exerted on each blade. According to this mechanism, the metallized cross-shaped microrotor can keep rotating while it is slightly tilted.

\section{Two-dimensional electromagnetic field analysis of radiation pressure}

To verify the driving mechanism, we estimated the optical force exerted on the cross-section of a blade by analyzing the two-dimensional electromagnetic field when a Gaussian laser beam is focused on the blade. In our simulation, we used commercial multiphysics analysis software (COMSOL ver. 4.1). The simulation model is shown in Fig. 8. In this model, a Gaussian laser beam (wavelength: $632 \mathrm{~nm}$; polarization: TM; beam waist: 0.699 $\mu \mathrm{m}$; peak electric field strength: $\left.1 \times 10^{6} \mathrm{~V} / \mathrm{m}\right)$ is incident on a rectangular polymer blade (width: $6 \mu \mathrm{m}$; height: $5 \mu \mathrm{m}$; refractive index: 1.52) coated with copper (thickness: 324 $\mathrm{nm}$; complex refractive index: $0.249+3.41$ i) in a liquid (refractive index: 1.41). The thickness of the copper layer is same as that of the copper layer observed by TEM as shown in Fig. 2 (c). The tilted angle of the metallized microrotor is $15^{\circ}$, which is the maximum tilt angle derived from the design of the microrotor. The origin $O$ of the $x$ coordinate is the center of the blade. The lateral position of the center of Gaussian beam is represented as the coordinate $P$ in $\mathrm{x}$ axis. Fig. 9 shows the optical intensity when the Gaussian beam is focused on the center of the blade $(P=0)$. The Gaussian beam is totally reflected at the bottom face of the tilted blade. Since the reflection causes a large momentum change in the incident photons, a large repulsive force is generated on the blade.

The net radiation pressure exerted on the blade is calculated using the Maxwell stress tensor. Fig. 10 shows the dependence of the net radiation pressure in lateral on focal position $P$. In this graph, the force towards to positive direction of $x$ axis is represented by positive value. In Fig. 10 , the blue and red lines show the results for the tilted and non-tilted blades, respectively. In both cases, the direction of the repulsive force is opposite at the two edges of the

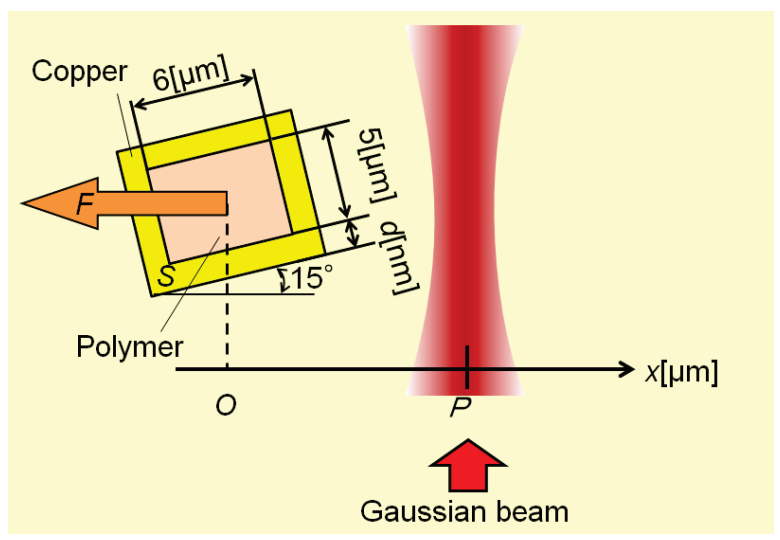

Fig. 8 Simulation model of radiation pressure exerted on a metallized blade tilted at $15^{\circ}$.

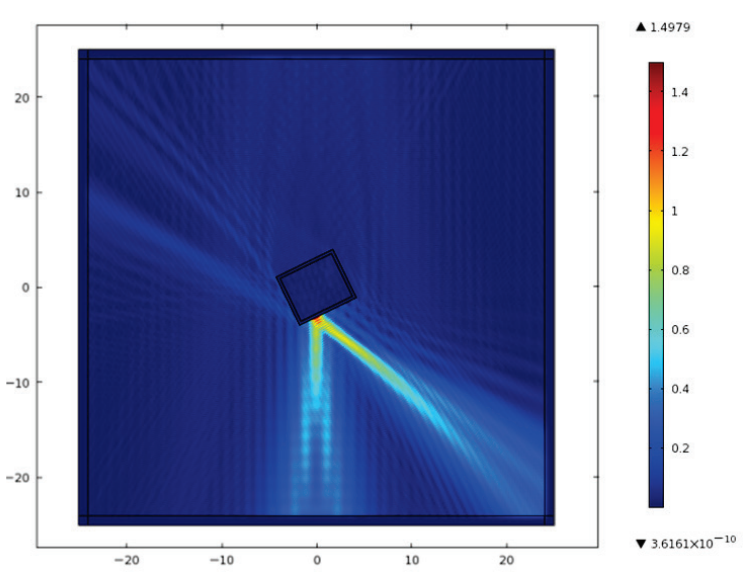

Fig. 9 Simulated electromagnetic field of a metallized microrotor blade when the Gaussian laser beam is focused on the center of the blade.

blade due to reflection at the metallic side. In the non-tilted blade, the forces generated at both edges are almost equal, so that the microrotor cannot rotate. On the other hand, in the case of the tilted blade, the total positive force is larger than the negative force owing to reflection at the bottom face. Therefore the tilted microrotor can be efficiently rotated by the total positive force applied to the blade when the laser beam passes through the blade.

\section{Conclusions}

We fabricated a cross-shaped metallized microrotor using two-photon microfabrication and subsequent 
electroless plating and laser ablation. The metallized microrotor could be rotated by scanning a low-power laser beam. The laser power required to drive the metallized

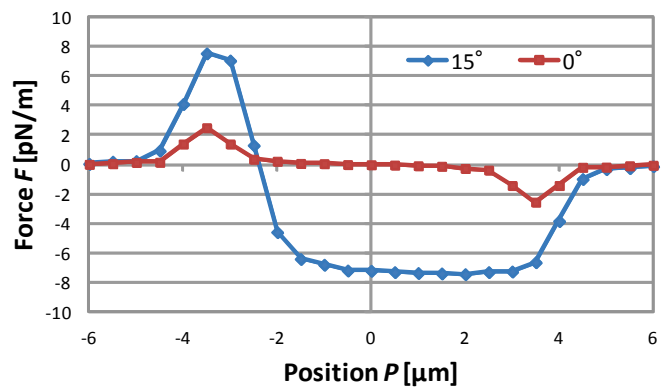

Fig. 10 Dependence of the lateral force exerted on metallized blades with tilt angles of 0 and 15 degrees. The red line represents the non-tilted blade and the blue line the tilted blade.

microrotor was less than one percent of that required for conventional polymeric microrotors, because the net repulsive force exerted on the metallic face of the blades is larger than attractive force applied to a dielectric microrotor. The reason why the metallized microrotor can be driven efficiently was investigated in detail by analyzing the radiation pressure exerted on the blade. It was confirmed that a large lateral force is generated on tilted blades. In the near future, highly-efficient optically-driven metallized micromachines will be developed by optimizing the topology of the blades. One of the promising applications of metallized micromachines is in low-cost, high-performance, lab-on-a-chip devices.

\section{Acknowledgments}

This research was partially supported by a Grant-in-Aid for Scientific Research (B) and a Grant-in-Aid for Challenging Exploratory Research from the Japan Society for the Promotion of Science. The authors would like to thank Prof. Kensuke Akamatsu, Konan University, for his cooperation in TEM observation of the cross section of the metallized copper layer.

\section{References}

[1] A. Ashkin, J.M. Dziedzic, and T. Yamane, "Optical trapping and manipulation of single cells using infrared laser beams," Nature 330, 769-71 (1987).

[2] A. Ashkin, "History of optical trapping and manipulation of small-neutral particle, atoms, and molecules,” IEEE J. Sel. Top, Quantum Electron. 6: 841-56 (2000).

[3] K.C. Neuman, and S.M. Block, "Optical trapping," Review of Scientific Instruments 75, 2787-2809 (2004).

[4] D.G. Grier, "A revolution in optical manipulation.," Nature 424, 810-816 (2003).

[5] A. Terray, J. Oakey, and D.W.M. Marr, "Microfluidic control using colloidal devices," Science 296, 1841-44 (2002).

[6] J. Leach, H. Mushfique, R. Leonardo, et al. "An optically driven pump for microfluidics," Lab Chip 6, 735-39 (2006).
[7] R.D. Leonardo, J. Leach, H. Mushfique, J.M. Cooper, G. Ruocco, and M.J. Padgett, "Multipoint Holographic Optical Velocimetry in Microfluidic Systems," Phys. Rev. Lett. 96, 134502 (2006).

[8] S. Maruo, O. Nakamura and S. Kawata, "Threedimensional microfabrication with two-photon absorbed photopolymerization," Opt. Lett. 22, 132-134 (1997).

[9] S. Kawata, H-B. Sun, T. Tanaka, and K. Takada, "Finer features for functional microdevices," Nature 412, 697-698 (2001).

[10] S. Maruo and H. Inoue, "Optically driven micropump produced by three-dimensional two-photon microfabrication,” Appl. Phys. Lett. 89, 144101 (2006)

[11] S. Maruo, A. Takaura, and Y. Saito, "Optically driven micropump with a twin spiral microrotor," Opt. Express 17, 18525-18532 (2009).

[12]C.-L. Lin, G. Vitrant, M. Bouriau, R. Casalegno, and P.L. Baldeck, "Optically driven Archimedes microscrews for micropump application," Opt. Express 19, 8267-8276 (2011).

[13] S. Maruo, K. Ikuta and H. Korogi, "Force-controllable, optically driven micromachines fabricated by singlestep two-photon microstereolithography," Journal of Microelectromech. Syst. 12, 533-539 (2003).

[14] C.-L. Lin, Y.-H. Lee, C.-T. Lin, Y.-J. Liu, J.-L. Hwang, T.-T. Chung, and P.L. Baldeck, "Multiplying optical tweezers force using a micro-lever," Opt. Express 19, 20604 -20609 (2011).

[15] K. Sasaki, M. Koshioka, H. Misawa, N. Kitamura, and H. Masuhara, "Optical trapping of a metal particle and a water droplet by a scanning laser beam," Appl. Phys. Lett. 60, 807-809 (1992).

[16]A.T. O'Neil and M.J. Padgett, "Three-dimensional optical confinement of micron-sized metal particles and the decoupling of the spin and orbital angular momentum within an optical spanner," Opt. Commun. 185, 139-143 (2000).

[17]M. Dienerowitz, M. Mazilu, P.J. Reece, T.F. Krauss and K. Dholakia, "Optical vortex trap for resonant confinement of metal nanoparticles," Opt. Express 16, 4991-4999 (2008).

[18] T. Ikegami, M. P. Stocker, K. Monaco, J. T. Fourkas, and S. Maruo, "Fabrication of three-dimensional metallized movable microstructures by the combination of two-photon microfabrication and electroless plating," Jpn. J. Appl. Phys. 51, 06FL17 (2012).

[19] R.A. Farrer, C.N. LaFratta, L. Li, J. Praino, M.J. Naughton, B.E.A. Saleh, M.C. Teich, J.T. Fourkas, "Selective Functionalization of 3-D Polymeric Microstructures," J. Am. Chem. Soc. 128, 1796 (2006).

[20] T. Baldacchini, C. LaFratta, R. A. Farrer, M. C. Teich, B. E. A. Saleh, M. J. Naughton and J. T. Fourkas, "Acrylic-Based Resin with Favorable Properties for Three-Dimensional Two-Photon Polymerization," J. Appl. Phys. 95, 6072 (2004).

(Received: August 02, 2012, Accepted: November 07, 2012) 